International Journal of Pure and Applied Mathematics

Volume 115 No. 3 2017, 497-515

ISSN: 1311-8080 (printed version); ISSN: 1314-3395 (on-line version)

url: http://www.ijpam.eu

doi: 10.12732/ijpam.v115i3.4

\title{
SOME COMMON FIXED POINT RESULTS FOR VARIOUS TYPES OF COMPATIBLE MAPPINGS IN MULTIPLICATIVE METRIC SPACES
}

Chahn Yong Jung ${ }^{1}$, Parveen Kumar ${ }^{2}$, Sanjay Kumar ${ }^{3}$, Shin Min Kang ${ }^{4}$

${ }^{1}$ Department of Business Administration

Gyeongsang National University

Jinju, 52828, KOREA

${ }^{2,3}$ Department of Mathematics

Deenbandhu Chhotu Ram University of Science and Technology

Murthal, Sonepat, 131039, Haryana, INDIA

${ }^{4}$ Department of Mathematics and RINS

Gyeongsang National University

Jinju, 52828, KOREA

\begin{abstract}
In this paper, we prove some common fixed point theorems for the mappings satisfying rational contractions along with various types of compatible mappings in multiplicative metric spaces.
\end{abstract}

AMS Subject Classification: 47H10, 54H 25

Key Words: multiplicative metric space, compatible, $A$-compatible of type $(E), S$-compatible of type $(E)$, weakly compatible, $A$-reciprocally continuous, $S$-reciprocally continuous

\section{Introduction and Preliminaries}

The set of positive real numbers $\mathbb{R}_{+}$is not complete according to the usual

Received: February 15, 2017

Revised: June 18, 2017

Published: July 27, 2017

(c) 2017 Academic Publications, Ltd. url: www.acadpubl.eu

${ }^{\S}$ Correspondence author 
metric. To overcome this problem, in 2008, Bashirov et al. [2] introduced the concept of multiplicative metric spaces as follows:

Definition 1.1. Let $X$ be a nonempty set. A multiplicative metric is a mapping $d: X \times X \rightarrow \mathbb{R}_{+}$satisfying the following conditions:

(i) $d(x, y) \geq 1$ for all $x, y \in X$ and $d(x, y)=1$ if and only if $x=y$;

(ii) $d(x, y)=d(y, x)$ for all $x, y \in X$;

(iii) $d(x, y) \leq d(x, z) \cdot d(z, y)$ for all $x, y, z \in X$ (multiplicative triangle inequality).

Then the mapping $d$ together with $X$, that is, $(X, d)$ is a multiplicative metric space.

Example 1.2. ([5]) Let $\mathbb{R}_{+}^{n}$ be the collection of all $n$-tuples of positive real numbers. Let $d^{*}: \mathbb{R}_{+}^{n} \times \mathbb{R}_{+}^{n} \rightarrow \mathbb{R}$ be defined as follows:

$$
d^{*}(x, y)=\left|\frac{x_{1}}{y_{1}}\right|^{*} \cdot\left|\frac{x_{2}}{y_{2}}\right|^{*} \cdots\left|\frac{x_{n}}{y_{n}}\right|^{*}
$$

where $x=\left(x_{1}, \ldots, x_{n}\right), y=\left(y_{1}, \ldots, y_{n}\right) \in \mathbb{R}_{+}^{n}$ and $|\cdot|^{*}: \mathbb{R}_{+} \rightarrow \mathbb{R}_{+}$is defined by

$$
|a|^{*}= \begin{cases}a & \text { if } a \geq 1, \\ \frac{1}{a} & \text { if } a<1 .\end{cases}
$$

Then it is obvious that all conditions of a multiplicative metric are satisfied. Therefore $\left(\mathbb{R}_{+}^{n}, d^{*}\right)$ is a multiplicative metric space.

Example 1.3. ([1]) Let $X=C^{*}[a, b]$ be the collection of all real-valued multiplicative continuous functions on $[a, b] \subset \mathbb{R}_{+}$. Then $(X, d)$ is a multiplicative metric space with $d$ defined by $d(f, g)=\sup _{x \in[a, b]}\left|\frac{f(x)}{g(x)}\right|$ for arbitrary $f, g \in X$.

Definition 1.4. ([5]) Let $(X, d)$ be a multiplicative metric space. Then a sequence $\left\{x_{n}\right\}$ in $X$ is said to be

(1) a multiplicative convergent to $x$ if for every multiplicative open ball $B_{\epsilon}(x)=\{y \mid d(x, y)<\epsilon\}, \epsilon>1$, there exists $N \in \mathbb{N}$ such that $x_{n} \in B_{\epsilon}(x)$ for all $n \geq N$, that is, $d\left(x_{n}, x\right) \rightarrow 1$ as $n \rightarrow \infty$.

(2) a multiplicative Cauchy sequence if for all $\epsilon>1$, there exists $N \in \mathbb{N}$ such that $d\left(x_{n}, x_{m}\right)<\epsilon$ for all $m, n \geq N$, that is, $d\left(x_{n}, x_{m}\right) \rightarrow 1$ as $n, m \rightarrow \infty$.

(3) We call a multiplicative metric space complete if every multiplicative Cauchy sequence in it is multiplicative convergent to $x \in X$.

In 2012, Özavsar and Çevikel [5] gave the concept of multiplicative contraction mappings and proved some fixed point theorems of such mappings in a 
multiplicative metric space.

Definition 1.5. Let $f$ be a mapping of a multiplicative metric space $(X, d)$ into itself. Then $f$ is said to be a multiplicative contraction if there exists a real number $\lambda \in[0,1)$ such that

$$
d(f x, f y) \leq d^{\lambda}(x, y) \quad \text { for all } x, y \in X .
$$

Recently, in 2015, Kang et al. [3, 4] introduced the notion of compatible and its variants, weakly compatible and reciprocally continuous mappings in multiplicative metric spaces as follows:

Definition 1.6. Let $A$ and $S$ be two mappings of a multiplicative metric space $(X, d)$ into itself. Then $A$ and $S$ are called

(1) compatible if

$$
\lim _{n \rightarrow \infty} d\left(A S x_{n}, S A x_{n}\right)=1
$$

whenever $\left\{x_{n}\right\}$ is a sequence in $X$ such that $\lim _{n \rightarrow \infty} A x_{n}=\lim _{n \rightarrow \infty} S x_{n}=t$ for some $t \in X$.

(2) compatible of type $(E)$ if

$$
\lim _{n \rightarrow \infty} A A x_{n}=\lim _{n \rightarrow \infty} A S x_{n}=S t
$$

and

$$
\lim _{n \rightarrow \infty} S S x_{n}=\lim _{n \rightarrow \infty} S A x_{n}=A t,
$$

whenever $\left\{x_{n}\right\}$ is a sequence in $X$ such that $\lim _{n \rightarrow \infty} A x_{n}=\lim _{n \rightarrow \infty} S x_{n}=t$ for some $t \in X$.

(3) reciprocally continuous if

$$
\lim _{n \rightarrow \infty} d\left(A S x_{n}, A t\right)=1 \text { and } \lim _{n \rightarrow \infty} d\left(S A x_{n}, S t\right)=1,
$$

whenever $\left\{x_{n}\right\}$ is a sequence in $X$ such that $\lim _{n \rightarrow \infty} A x_{n}=\lim _{n \rightarrow \infty} S x_{n}=t$ for some $t \in X$.

(4) weakly compatible if they commute at coincidence points, that is, $A x=$ $S x$ for $x \in X$ implies $A S x=S A x$.

\section{Various Types of Compatible Mappings and their Relationships}

In 2011, Singh and Singh [7] introduced the notions of compatible of type $(E)$, $A$-compatible of type $(E)$ and $S$-compatible of type $(E)$. 
In 1999, Pant [6] introduced the concept of reciprocally continuous mappings. Also, Singh and Singh [7] introduced the notions of reciprocally continuous into $A$-reciprocally continuous and $S$-reciprocally continuous.

Now, we introduce these notions in setting of multiplicative metric spaces.

Definition 2.1. Let $A$ and $S$ be two mappings of a multiplicative metric space $(X, d)$ into itself. Then $A$ and $S$ are called

(1) $S$-compatible of type $(E)$ if

$$
\lim _{n \rightarrow \infty} S S x_{n}=\lim _{n \rightarrow \infty} S A x_{n}=A t,
$$

whenever $\left\{x_{n}\right\}$ is a sequence in $X$ such that $\lim _{n \rightarrow \infty} A x_{n}=\lim _{n \rightarrow \infty} S x_{n}=t$ for some $t \in X$.

(2) A-compatible of type (E) if

$$
\lim _{n \rightarrow \infty} A A x_{n}=\lim _{n \rightarrow \infty} A S x_{n}=S t,
$$

whenever $\left\{x_{n}\right\}$ is a sequence in $X$ such that $\lim _{n \rightarrow \infty} A x_{n}=\lim _{n \rightarrow \infty} S x_{n}=t$ for some $t \in X$.

(3) S-reciprocally continuous if

$$
\lim _{n \rightarrow \infty} S A x_{n}=S t,
$$

whenever $\left\{x_{n}\right\}$ is a sequence in $X$ such that $\lim _{n \rightarrow \infty} A x_{n}=\lim _{n \rightarrow \infty} S x_{n}=t$ for some $t \in X$.

(4) A-reciprocally continuous if

$$
\lim _{n \rightarrow \infty} A S x_{n}=A t,
$$

whenever $\left\{x_{n}\right\}$ is a sequence in $X$ such that $\lim _{n \rightarrow \infty} A x_{n}=\lim _{n \rightarrow \infty} S x_{n}=t$ for some $t \in X$.

Remark 2.2. 1. It is easy to see that compatible of type $(E)$ implies both $A$-compatible of type $(E)$ and $S$-compatible of type $(E)$, however $A$-compatible of type $(E)$ or $S$-compatible of type $(E)$ do not imply compatible of type $(E)$ (see Example 2.3)

2. Clearly, the reciprocal continuity of $A, S$ implies both $A$-reciprocal continuity and $S$-reciprocal continuity, however $A$-reciprocal contionuity or $S$ reciprocal continuity do not imply reciprocal continuity (see Example 2.4). 
Example 2.3. Let $X=[0,1]$ and $(X, d)$ be a complete multiplicative metric space, where $d$ is defined by $d(x, y)=e^{|x-y|}$ for all $x, y \in X$. Let $A$ and $S: X \rightarrow X$ be two mappings defined by

$$
A x=\left\{\begin{array}{ll}
1 & \text { if } x \in\left[0, \frac{1}{2}\right]-\left\{\frac{1}{4}\right\}, \\
0 & \text { if } x=\frac{1}{4}, \\
\frac{1-x}{2} & \text { if } x \in\left(\frac{1}{2}, 1\right],
\end{array} \quad S x= \begin{cases}\frac{1}{5} \quad \text { if } x \in\left[0, \frac{1}{2}\right]-\left\{\frac{1}{4}\right\} \\
1 \quad \text { if } x=\frac{1}{4} \\
\frac{x}{2} \quad \text { if } x \in\left(\frac{1}{2}, 1\right] .\end{cases}\right.
$$

Since $A$ and $S$ are not continuous at $x=\frac{1}{2}, \frac{1}{4}$. Suppose that $x_{n} \rightarrow \frac{1}{2}$, $x_{n}>\frac{1}{2}$ for all $n$. Then, we have $A x_{n}=\frac{1-x_{n}}{2} \rightarrow \frac{1}{4}=t$ and $S x_{n}=\frac{x_{n}}{2} \rightarrow \frac{1}{4}=t$. Consequently, we have $A A x_{n}=A\left(\frac{1-x_{n}}{2}\right) \rightarrow 1, A S x_{n}=A\left(\frac{x_{n}}{2}\right) \rightarrow 1, S(t)=1$ and $S S x_{n}=S\left(\frac{x_{n}}{2}\right) \rightarrow \frac{1}{5}, S A x_{n}=S\left(\frac{1-x_{n}}{2}\right) \rightarrow \frac{1}{5}, A(t)=0$. Hence the pair $A$ and $S$ are $A$-compatible of type (E) but not compatible of type $(E)$ because it is not $S$-compatible of type $(E)$.

Example 2.4. Let $X=[0,1]$ and $(X, d)$ be a complete multiplicative metric space, where $d$ is defined by $d(x, y)=e^{|x-y|}$ for all $x, y \in X$. Let $A, S$ : $X \rightarrow X$ be two mappings defined by

$$
A x=\left\{\begin{array}{ll}
1 & \text { if } x \in\left[0, \frac{1}{2}\right), \\
1-x & \text { if }\left[\frac{1}{2}, 1\right], \\
\frac{1-x}{2} & \text { if } x \in\left(\frac{1}{2}, 1\right],
\end{array} \quad S x= \begin{cases}0 & \text { if } x \in\left[0, \frac{1}{2}\right) \\
x & \text { if }\left[\frac{1}{2}, 1\right] \\
x / 2 & \text { if } x \in\left(\frac{1}{2}, 1\right]\end{cases}\right.
$$

Since $A$ and $S$ are not continuous at $x=\frac{1}{2}, \frac{1}{4}$. Suppose that $x_{n} \rightarrow \frac{1}{2}$, $x_{n}>\frac{1}{2}$ for all $n$. Then, we have $A x_{n}=1-x_{n} \rightarrow \frac{1}{2}=t$ and $S x_{n}=x_{n} \rightarrow$ $\frac{1}{2}=t$. Consequently, we have $A S x_{n}=A\left(x_{n}\right)=1-x_{n} \rightarrow \frac{1}{2}, A(t)=\frac{1}{2}$, $S A x_{n}=S\left(1-x_{n}\right) \rightarrow 0, S(t)=\frac{1}{2}$. It follows that $\lim _{n \rightarrow \infty} A S x_{n}=\frac{1}{2}=$ $A(t)$ and $\lim _{n \rightarrow \infty} S A x_{n}=0 \neq \frac{1}{2}=S(t)$. Therefore, the pair $A$ and $S$ are $A$ reciprocally continuous. However the pair is neither $S$-reciprocally continuous nor reciprocally continuous.

Proposition 2.5. Let $A$ and $S$ be two mappings of a multiplicative metric space $(X, d)$ into itself. Suppose that $\left\{x_{n}\right\}$ is a sequence in $X$ such that $A x_{n} \rightarrow$ and $S x_{n} \rightarrow t$ for some $t \in X$. If one of the following conditions is satisfied:

$(a)$ the pair $A$ and $S$ are $S$-compatible of type $(E)$ and $S$-reciprocally continuous;

(b) the pair $A$ and $S$ are $A$-compatible of type $(E)$ and $A$-reciprocally continuous.

Then $A t=S t$ and if there exists $u \in X$ such that $A u=S u=t$, then $A S u=S A u$. 
Proof. Assume that $(a)$ hold and $\left\{x_{n}\right\}$ is a sequence in $X$ such that $A x_{n} \rightarrow$ and $S x_{n} \rightarrow t$. Then $\lim _{n \rightarrow \infty} S S x_{n}=\lim _{n \rightarrow \infty} S A x_{n}=A t$ and $\lim _{n \rightarrow \infty} S A x_{n}=$ $S t$ implies that $A t=S t$. Now if there exists $u \in X$ such that $A u=S u=t$, then $A S u=A t=S t=S A u$.

Similarly, assume that $(b)$ hols, we oatain the same result.

\section{Main Results}

Now we prove the following theorem for mappings satisfying rational contraction along with compatible and weakly compatible mappings as follows:

Theorem 3.1. Let $A, B, S$ and $T$ be mappings of a complete multiplicative metric space $(X, d)$ into itself satisfying the following conditions: $S X \subset B X, \quad T X \subset A X$

$$
d(S x, T y) \leq[\varphi(M(x, y))]^{\lambda}
$$

for each $x, y \in X$ and $\lambda \in(0,1)$, where $\varphi:[1, \infty) \rightarrow[1, \infty)$ is a monotone increasing continuous function such that $\varphi(1)=1, \varphi(t)<t$ for all $t>1$, $\varphi(t)=\sqrt{2 t-1}$ for all $t \geq 1$ and

$$
\begin{aligned}
M(x, y)=\max \{d(A x, B y), d(A x, S x), d(B y, T y), & (d(A x, T y) \cdot d(S x, B y))^{1 / 2}, \\
& \frac{d(A x, T y) \cdot d(S x, B y)}{1+d(A x, B y)}, \frac{d(A x, S x) \cdot d(B y, T y)}{1+d(A x, B y)}, \\
& \left.\frac{1+d(A x, T y) \cdot d(B y, S x)}{1+d(A x, S x) \cdot d(B y, T y)} \cdot d(A x, S x)\right\} .
\end{aligned}
$$

Suppose that one of the following conditions is satisfied:

(a) either $A$ or $S$ is continuous, $A$ and $S$ are compatible, and $B$ and $T$ are weakly compatible.

(b) either $B$ or $T$ is continuous, $B$ and $T$ are compatible, and $A$ and $S$ are weakly compatible.

Then $A, B, S$ and $T$ have a unique common fixed point. 
Proof. Let $x_{0} \in X$ be an arbitrary point. Since $S X \subset B X$, for point $x_{0} \in X$, there exists a point $x_{1} \in X$ such that $S x_{0}=B x_{1}$. Now for this point $x_{1}$ there exists a point $x_{2} \in X$ such that $T x_{1}=A x_{2}=y_{1}$. Similarly, we can inductively define a sequence $\left\{y_{n}\right\}$ such that

$$
S x_{2 n}=B x_{2 n+1}=y_{2 n} ; \quad T x_{2 n+1}=A x_{2 n+2}=y_{2 n+1} \quad \text { for } n \geq 0 .
$$

Now from $\left(C_{2}\right)$, we obtain

$$
d\left(y_{2 n}, y_{2 n+1}\right)=d\left(S x_{2 n}, T x_{2 n+1}\right) \leq \varphi\left(M\left(x_{2 n}, x_{2 n+1}\right)\right)^{\lambda}
$$

where by the multiplicative triangle inequality

$$
\begin{aligned}
& M\left(x_{2 n}, x_{2 n+1}\right) \max \left\{d\left(A x_{2 n}, B x_{2 n+1}\right), d\left(A x_{2 n}, S x_{2 n}\right), d\left(B x_{2 n+1}, T x_{2 n+1}\right)\right. \\
&\left(d\left(A x_{2 n}, T x_{2 n+1}\right) \cdot d\left(S x_{2 n}, B x_{2 n+1}\right)\right)^{1 / 2}, \\
& \frac{d\left(A x_{2 n}, T x_{2 n+1}\right) \cdot d\left(S x_{2 n}, B x_{2 n+1}\right)}{1+d\left(A x_{2 n}, B x_{2 n+1}\right)}, \frac{d\left(A x_{2 n}, S x_{2 n}\right) \cdot d\left(B x_{2 n+1}, T x_{2 n+1}\right)}{1+d\left(A x_{2 n}, B x_{2 n+1}\right)}, \\
&\left.\frac{1+d\left(A x_{2 n}, T x_{2 n+1}\right) \cdot d\left(B x_{2 n+1}, S x_{2 n}\right)}{1+d\left(A x_{2 n}, S x_{2 n}\right) \cdot d\left(B x_{2 n+1}, T x_{2 n+1}\right)} \cdot d\left(A x_{2 n}, S x_{2 n}\right)\right\} \\
&= \max \left\{d\left(y_{2 n-1}, y_{2 n}\right), d\left(y_{2 n-1}, y_{2 n}\right), d\left(y_{2 n}, y_{2 n+1}\right),\right. \\
&\left(d\left(y_{2 n-1}, y_{2 n+1}\right) \cdot d\left(y_{2 n}, y_{2 n}\right)\right)^{1 / 2}, \\
& \frac{d\left(y_{2 n-1}, y_{2 n+1}\right) \cdot d\left(y_{2 n}, y_{2 n}\right)}{1+d\left(y_{2 n-1}, y_{2 n}\right)}, \frac{d\left(y_{2 n-1}, y_{2 n}\right) \cdot d\left(y_{2 n}, y_{2 n+1}\right)}{1+d\left(y_{2 n-1}, y_{2 n}\right)}, \\
&\left.\frac{1+d\left(y_{2 n-1}, y_{2 n+1}\right) \cdot d\left(y_{2 n}, y_{2 n}\right)}{1+d\left(y_{2 n-1}, y_{2 n}\right) \cdot d\left(y_{2 n}, y_{2 n+1}\right)} \cdot d\left(y_{2 n-1}, y_{2 n}\right)\right\} \\
&= \max \left\{d\left(y_{2 n-1}, y_{2 n}\right), d\left(y_{2 n}, y_{2 n+1}\right),\left(d\left(y_{2 n-1}, y_{2 n}\right) \cdot d\left(y_{2 n}, y_{2 n+1}\right)\right)^{1 / 2}\right. \\
& \frac{d\left(y_{2 n-1}, y_{2 n}\right) \cdot d\left(y_{2 n}, y_{2 n+1}\right)}{1+d\left(y_{2 n-1}, y_{2 n}\right)}, \frac{d\left(y_{2 n-1}, y_{2 n}\right) \cdot d\left(y_{2 n}, y_{2 n+1}\right)}{1+d\left(y_{2 n-1}, y_{2 n}\right)}, \\
&\left.\frac{1+d\left(y_{2 n-1}, y_{2 n}\right) \cdot d\left(y_{2 n}, y_{2 n+1}\right)}{1+d\left(y_{2 n-1}, y_{2 n}\right) \cdot d\left(y_{2 n}, y_{2 n+1}\right)} d\left(y_{2 n-1}, y_{2 n}\right)\right\} \\
& \max \left\{d\left(y_{2 n-1}, y_{2 n}\right), d\left(y_{2 n}, y_{2 n+1}\right),\left(d\left(y_{2 n-1}, y_{2 n}\right) \cdot d\left(y_{2 n}, y_{2 n+1}\right)\right)^{1 / 2}\right\} . \\
&=
\end{aligned}
$$


If $d\left(y_{2 n-1}, y_{2 n}\right)<d\left(y_{2 n}, y_{2 n+1}\right)$, then we have

$$
\begin{aligned}
d\left(y_{2 n}, y_{2 n+1}\right) & =d\left(S x_{2 n}, T x_{2 n+1}\right) \\
& \leq\left[\varphi\left(M\left(x_{2 n}, x_{2 n+1}\right)\right)\right]^{\lambda} \\
& =\left[\varphi\left(d\left(y_{2 n}, y_{2 n+1}\right)\right)\right]^{\lambda} \\
& <d^{\lambda}\left(y_{2 n}, y_{2 n+1}\right)
\end{aligned}
$$

which is a contraction. Hence $d\left(y_{2 n}, y_{2 n+1}\right) \leq d\left(y_{2 n-1}, y_{2 n}\right)$ and $M\left(x_{2 n}, x_{2 n+1}\right)=$ $d\left(y_{2 n-1}, y_{2 n}\right)$. Thus we have

$$
d\left(y_{2 n}, y_{2 n+1}\right)<d^{\lambda}\left(y_{2 n-1}, y_{2 n}\right)
$$

where $\lambda \in(0,1)$.

Similarly, we have

$$
d\left(y_{2 n+2}, y_{2 n+1}\right)=d\left(S x_{2 n+2}, T x_{2 n+1}\right) \leq\left[\varphi\left(M\left(x_{2 n+2}, x_{2 n+1}\right)\right)\right]^{\lambda},
$$

where

$$
\begin{aligned}
& M(\left.x_{2 n+2}, x_{2 n+1}\right) \\
&= \max \left\{d\left(A x_{2 n+2}, B x_{2 n+1}\right), d\left(A x_{2 n+2}, S x_{2 n+2}\right), d\left(B x_{2 n+1}, T x_{2 n+1}\right),\right. \\
&\left(d\left(A x_{2 n+2}, T x_{2 n+1}\right) \cdot d\left(S x_{2 n+2}, B x_{2 n+1}\right)\right)^{1 / 2}, \\
& \frac{d\left(A x_{2 n+2}, T x_{2 n+1}\right) \cdot d\left(S x_{2 n+2}, B x_{2 n+1}\right)}{1+d\left(A x_{2 n+2}, B x_{2 n+1}\right)}, \\
& \frac{d\left(A x_{2 n+2}, S x_{2 n+2}\right) \cdot d\left(B x_{2 n+1}, T x_{2 n+1}\right)}{1+d\left(A x_{2 n+2}, B x_{2 n+1}\right)}, \\
&\left.\frac{1+d\left(A x_{2 n+2}, T x_{2 n+1}\right) \cdot d\left(B x_{2 n+1}, S x_{2 n+2}\right)}{1+d\left(A x_{2 n+2}, S x_{2 n+2}\right) \cdot d\left(B x_{2 n+1}, T x_{2 n+1}\right)} \cdot d\left(A x_{2 n+2}, S x_{2 n+2}\right)\right\} \\
&=\max \left\{d\left(y_{2 n+1}, y_{2 n}\right), d\left(y_{2 n+1}, y_{2 n+2}\right), d\left(y_{2 n}, y_{2 n+1}\right),\right. \\
&\left(d\left(y_{2 n+1}, y_{2 n+1}\right) \cdot d\left(y_{2 n+2}, y_{2 n}\right)\right)^{1 / 2}, \\
& \frac{d\left(y_{2 n+1}, y_{2 n+1}\right) \cdot d\left(y_{2 n+2}, y_{2 n}\right)}{1+d\left(y_{2 n+1}, y_{2 n}\right)}, \frac{d\left(y_{2 n+1}, y_{2 n+2}\right) \cdot d\left(y_{2 n}, y_{2 n+1}\right)}{1+d\left(y_{2 n+1}, y_{2 n}\right)}, \\
&\left.\frac{1+d\left(y_{2 n+1}, y_{2 n+1}\right) \cdot d\left(y_{2 n}, y_{2 n+2}\right)}{1+d\left(y_{2 n+1}, y_{2 n+2}\right) \cdot d\left(y_{2 n}, y_{2 n+1}\right)} \cdot d\left(y_{2 n+1}, y_{2 n+2}\right)\right\} \\
&=\max \left\{d\left(y_{2 n+1}, y_{2 n}\right), d\left(y_{2 n+1}, y_{2 n+2}\right),\left(d\left(y_{2 n}, y_{2 n+1}\right) \cdot d\left(y_{2 n+2}, y_{2 n+1}\right)\right)^{1 / 2}\right\} .
\end{aligned}
$$


If $d\left(y_{2 n+1}, y_{2 n}\right) \leq d\left(y_{2 n+1}, y_{2 n+2}\right)$, then we have

$$
\begin{aligned}
d\left(y_{2 n+2}, y_{2 n+1}\right) & =d\left(S x_{2 n}, T x_{2 n+1}\right) \\
& \leq\left[\varphi\left(d\left(y_{2 n+1}, y_{2 n+2}\right)\right)\right]^{\lambda} \\
& <d^{\lambda}\left(y_{2 n+1}, y_{2 n+2}\right),
\end{aligned}
$$

which is a contraction. Hence we have

$$
d\left(y_{2 n+2}, y_{2 n+1}\right)<d^{\lambda}\left(y_{2 n+1}, y_{2 n}\right)
$$

where $\lambda \in(0,1)$.

It follows from (3.1) and (3.2) that, for all $n \in \mathbb{N}$,

$$
\begin{aligned}
d\left(y_{n}, y_{n+1}\right) & \leq d^{\lambda}\left(y_{n-1}, y_{n}\right) \\
& \leq d^{\lambda^{2}}\left(y_{n-2}, y_{n-1}\right) \\
& \leq \cdots \leq d^{\lambda^{n}}\left(y_{0}, y_{1}\right)
\end{aligned}
$$

Let $m, n \in \mathbb{N}$ with $m>n$, we get

$$
\begin{aligned}
d\left(y_{m}, y_{n}\right) & \leq d\left(y_{m}, y_{m-1}\right) \cdots d\left(y_{n+1}, y_{n}\right) \\
& \leq d^{\lambda^{m-1}+\cdots+\lambda^{n}}\left(y_{1}, y_{0}\right) \\
& \leq d^{\frac{\lambda^{n}}{1-\lambda}}\left(y_{1}, y_{0}\right) \\
& \rightarrow 1 \quad \text { as } m, n \rightarrow \infty
\end{aligned}
$$

Hence $\left\{y_{n}\right\}$ is a multiplicative Cauchy sequence. Since $X$ is complete so $\left\{y_{n}\right\} \rightarrow$ $z \in X$. Therefore, subsequences $\left\{S x_{2 n}\right\},\left\{B x_{2 n+1}\right\},\left\{A x_{2 n}\right\}$ and $\left\{T x_{2 n+1}\right\}$ also converges to $z \in X$. Thus we get

$$
\lim _{n \rightarrow \infty} S x_{2 n}=\lim _{n \rightarrow \infty} B x_{2 n+1}=\lim _{n \rightarrow \infty} A x_{2 n}=\lim _{n \rightarrow \infty} T x_{2 n+1}=z .
$$

Next, we show that $z$ is a common fixed point of $A, B, S$ and $T$ under the condition $(a)$.

Case 1. Suppose that $A$ is continuous. Then it follows that $\lim _{n \rightarrow \infty} A S x_{2 n}=$ $\lim _{n \rightarrow \infty} A A x_{2 n}=A z$. Since $A$ and $S$ are compatible, it follows from (3.3) that

$$
\lim _{n \rightarrow \infty} d\left(S A x_{2 n}, A z\right)=\lim _{n \rightarrow \infty} d\left(S A x_{2 n}, A S x_{2 n}\right)=1
$$

this is, $\lim _{n \rightarrow \infty} S A x_{2 n}=A z$. 
First we claim that $A z=z$. Let $A z \neq z$. Then putting $x=A x_{2 n}$ and $y=x_{2 n+1}$ in inequality $\left(C_{2}\right)$, we get

$$
d\left(S A x_{2 n}, T x_{2 n+1}\right) \leq\left[\varphi\left(M\left(A x_{2 n}, x_{2 n+1}\right)\right)\right]^{\lambda}
$$

where

$$
\begin{aligned}
M( & \left.A x_{2 n}, x_{2 n+1}\right) \\
= & \max \left\{d\left(A A x_{2 n}, B x_{2 n+1}\right), d\left(A A x_{2 n}, S A x_{2 n}\right), d\left(B x_{2 n+1}, T x_{2 n+1}\right),\right. \\
& \left(d\left(A A x_{2 n}, T x_{2 n+1}\right) \cdot d\left(S A x_{2 n}, B x_{2 n+1}\right)\right)^{1 / 2}, \\
& \frac{d\left(A A x_{2 n}, T x_{2 n+1}\right) \cdot d\left(S A x_{2 n}, B x_{2 n+1}\right)}{1+d\left(A A x_{2 n}, B x_{2 n+1}\right)}, \\
& \frac{d\left(A A x_{2 n}, S A x_{2 n}\right) \cdot d\left(B x_{2 n+1}, T x_{2 n+1}\right)}{1+d\left(A A x_{2 n}, B x_{2 n+1}\right)}, \\
& \left.\frac{1+d\left(A A x_{2 n}, T x_{2 n+1}\right) \cdot d\left(B x_{2 n+1}, S A x_{2 n}\right)}{1+d\left(A A x_{2 n}, S A x_{2 n}\right) \cdot d\left(B x_{2 n+1}, T x_{2 n+1}\right)} \cdot d\left(A A x_{2 n}, S A x_{2 n}\right)\right\} .
\end{aligned}
$$

Taking $n \rightarrow \infty$, we have

$$
\begin{aligned}
\lim _{n \rightarrow \infty} M\left(A x_{2 n}, x_{2 n+1}\right) & \\
= & \max \left\{d(A z, z), 1,1, d(A z, z), \frac{d(A z, z) \cdot d(A z, z)}{1+d(A z, z)},\right. \\
& \left.\frac{1}{1+d(A z, z)}, \frac{1+d(A z, z) \cdot d(z, A z)}{2}\right\} \\
= & \frac{1+d^{2}(z, A z)}{2} .
\end{aligned}
$$

Hence we have

$$
d(A z, z) \leq\left[\varphi\left(\frac{1+d^{2}(z, A z)}{2}\right)\right]^{\lambda} \leq d^{\lambda}(z, A z)
$$

by property $\varphi$, which implies that $A z=z$.

Next we claim that $S z=z$. Let $S z \neq z$. Then putting $x=z$ and $y=x_{2 n+1}$ in inequality $\left(C_{2}\right)$, we have

$$
d\left(S z, T x_{2 n+1}\right) \leq\left[\varphi\left(M\left(z, x_{2 n+1}\right)\right)\right]^{\lambda},
$$


where

$$
\begin{aligned}
M\left(z, x_{2 n+1}\right) & \max \left\{d\left(A z, B x_{2 n+1}\right), d(A z, S z), d\left(B x_{2 n+1}, T x_{2 n+1}\right),\right. \\
& \left(d\left(A z, T x_{2 n+1}\right) \cdot d\left(S z, B x_{2 n+1}\right)\right)^{1 / 2}, \\
& \frac{d\left(A z, T x_{2 n+1}\right) \cdot d\left(S z, B x_{2 n+1}\right)}{1+d\left(A z, B x_{2 n+1}\right)}, \frac{d(A z, S z) \cdot d\left(B x_{2 n+1}, T x_{2 n+1}\right)}{1+d\left(A z, B x_{2 n+1}\right)}, \\
& \left.\frac{1+d\left(A z, T x_{2 n+1}\right) \cdot d\left(B x_{2 n+1}, S z\right)}{1+d(A z, S z) \cdot d\left(B x_{2 n+1}, T x_{2 n+1}\right)} \cdot d(A z, S z)\right\} .
\end{aligned}
$$

Taking $n \rightarrow \infty$ and using $A z=z$, we have

$$
\begin{aligned}
& \lim _{n \rightarrow \infty} M\left(z, x_{2 n+1}\right) \\
& =\max \left\{1, d(z, S z), 1, d^{1 / 2}(S z, z), \frac{d(z, S z)}{2}, \frac{d(z, S z)}{2}, d(z, S z)\right\} \\
& =d(z, S z) .
\end{aligned}
$$

Hence we have

$$
d(S z, z)=\lim _{n \rightarrow \infty} d\left(S z, T x_{2 n+1}\right) \leq[\varphi(d(z, S z))]^{\lambda}<d^{\lambda}(z, S z),
$$

which implies that $S z=z$.

On the other hand, since $z=S z \in S(X) \subset B(X)$, there exist $u \in X$ such that $z=S z=B u$.

Next we claim that $T u=z$. Let $T u \neq z$. Then using $z=S z=A z=B u$ and putting $x=z$ and $y=u$ in inequality $\left(C_{2}\right)$, we have

$$
d(z, T u)=d(S z, T u) \leq[\varphi(M(z, u))]^{\lambda},
$$

where

$$
\begin{aligned}
& M(z, u)=\max \{d(A z, B u), d(A z, S z), d(B u, T u) \\
&(d(A z, T u) \cdot d(S z, B u))^{1 / 2}, \\
& \frac{d(A z, T u) \cdot d(S z, B u)}{1+d(A z, B u)}, \frac{d(A z, S z) \cdot d(B u, T u)}{1+d(A z, B u)}, \\
&\left.\frac{1+d(A z, T u) \cdot d(B u, S z)}{1+d(A z, S z) \cdot d(B u, T u)} \cdot d(A z, S z)\right\} \\
&= \max \left\{1,1, d(z, T u), d^{1 / 2}(z, T u), \frac{d(z, T u)}{2}, \frac{d(z, T u)}{2}, 1\right\} \\
&= d(z, T u),
\end{aligned}
$$


so $T u=z=B u$. Since $B$ an $T$ are weakly compatible, we have $T z=T B u=$ $B T u=B z$.

Next, we prove that $T z=z$. Putting $x=z$ and $y=z$ in inequality $\left(C_{2}\right)$, we have

$$
d(z, T z)=d(S z, T z) \leq[\varphi(M(z, z))]^{\lambda}
$$

where

$$
\begin{aligned}
M(z, z)= & \max \{d(A z, B z), d(A z, S z), d(B z, T z), \\
& (d(A z, T z) \cdot d(S z, B z))^{1 / 2}, \\
& \frac{d(A z, T z) \cdot d(S z, B z)}{1+d(A z, B z)}, \frac{d(A z, S z) \cdot d(B z, T z)}{1+d(A z, B z))}, \\
& \left.\frac{1+d(A z, T z) \cdot d(B z, S z)}{1+d(A z, S z) \cdot d(B z, T z)} \cdot d(A z, S z)\right\} \\
= & \max \left\{1,1, d(z, T z), d^{1 / 2}(z, T z), \frac{d(z, T z)}{2}, \frac{d(z, T z)}{2}, 1\right\} \\
= & d(z, T z) .
\end{aligned}
$$

This implies that $d(z, T z)=1$ and so $z=T z$. Hence we obtain $z=S z=A z=$ $T z=B z$ and hence $z$ is a common fixed point of $A, B, S$ and $T$.

Case 2. Suppose that $S$ is continuous. Then it follows that $\lim _{n \rightarrow \infty} S A x_{2 n}=$ $\lim _{n \rightarrow \infty} S S x_{2 n}=S z$. Since $A$ and $S$ are compatible, it follows from (3.3) that

$$
\lim _{n \rightarrow \infty} d\left(S z, A S x_{2 n}\right)=\lim _{n \rightarrow \infty} d\left(S A x_{2 n}, A S x_{2 n}\right)=1
$$

this is, $\lim _{n \rightarrow \infty} A S x_{2 n}=S z$.

First we claim that $S z=z$. Let $S z \neq z$. Then putting $x=S x_{2 n}$ and $y=x_{2 n+1}$ in inequality $\left(C_{2}\right)$, we get

$$
d\left(S S x_{2 n}, T x_{2 n+1}\right) \leq\left[\varphi\left(M\left(S x_{2 n}, x_{2 n+1}\right)\right)\right]^{\lambda},
$$


where

$$
\begin{aligned}
M( & \left.S x_{2 n}, x_{2 n+1}\right) \\
= & \max \left\{d\left(A S x_{2 n}, B x_{2 n+1}\right), d\left(A S x_{2 n}, S S x_{2 n}\right), d\left(B x_{2 n+1}, T x_{2 n+1}\right),\right. \\
& \left(d\left(A S x_{2 n}, T x_{2 n+1}\right) \cdot d\left(S S x_{2 n}, B x_{2 n+1}\right)\right)^{1 / 2}, \\
& \frac{d\left(A S x_{2 n}, T x_{2 n+1}\right) \cdot d\left(S S x_{2 n}, B x_{2 n+1}\right)}{1+d\left(A S x_{2 n}, B x_{2 n+1}\right)}, \\
& \frac{d\left(A S x_{2 n}, S S x_{2 n}\right) \cdot d\left(B x_{2 n+1}, T x_{2 n+1}\right)}{1+d\left(A S x_{2 n}, B x_{2 n+1}\right)}, \\
& \left.\frac{1+d\left(A S x_{2 n}, T x_{2 n+1}\right) \cdot d\left(B x_{2 n+1}, S S x_{2 n}\right)}{1+d\left(A S x_{2 n}, S S x_{2 n}\right) \cdot d\left(B x_{2 n+1}, T x_{2 n+1}\right)} \cdot d\left(A S x_{2 n}, S S x_{2 n}\right)\right\} .
\end{aligned}
$$

Taking $n \rightarrow \infty$, we have

$$
\begin{aligned}
\lim _{n \rightarrow \infty} M\left(S x_{2 n}, x_{2 n+1}\right) \\
=\max \left\{d(S z, z), 1,1, d(S z, z), \frac{d(S z, z) \cdot d(S z, z)}{1+d(S z, z)}\right. \\
\left.\quad \frac{1}{1+d(S z, z)}, \frac{1+d(S z, z) \cdot d(z, S z)}{2}\right\} \\
=\frac{1+d^{2}(z, S z)}{2} .
\end{aligned}
$$

Hence we have

$$
d(S z, z) \leq\left[\varphi\left(\frac{1+d^{2}(z, S z)}{2}\right)\right]^{\lambda} \leq d^{\lambda}(z, S z)
$$

which implies that $S z=z$. Since $z=S z \in S(X) \subset B(X)$, there exist $v \in X$ such that $z=S z=B v$.

Next we claim that $T v=z$. Let $T v \neq z$. Then putting $x=S x_{2 n}$ and $y=v$ in inequality $\left(C_{2}\right)$, we have

$$
d\left(S S x_{2 n}, T v\right) \leq\left[\varphi\left(M\left(S x_{2 n}, v\right)\right)\right]^{\lambda}
$$


where

$$
\begin{aligned}
M( & \left.S x_{2 n}, v\right) \\
= & \max \left\{d\left(A S x_{2 n}, B v\right), d\left(A S x_{2 n}, S S x_{2 n}\right), d(B v, T v),\right. \\
& \left(d\left(A S x_{2 n}, T v\right) \cdot d\left(S S x_{2 n}, B v\right)\right)^{1 / 2} \\
& \frac{d\left(A S x_{2 n}, T v\right) \cdot d\left(S S x_{2 n}, B v\right)}{1+d\left(A S x_{2 n}, B v\right)}, \frac{d\left(A S x_{2 n}, S S x_{2 n}\right) \cdot d(B v, T v)}{1+d\left(A S x_{2 n}, B v\right)}, \\
& \left.\frac{1+d\left(A S x_{2 n}, T v\right) \cdot d\left(B v, S S x_{2 n}\right)}{1+d\left(A S x_{2 n}, S S x_{2 n}\right) \cdot d(B v, T v)} \cdot d\left(A S x_{2 n}, S S x_{2 n}\right)\right\}
\end{aligned}
$$

Taking $n \rightarrow \infty$, we have

$$
\begin{aligned}
& \lim _{n \rightarrow \infty} M\left(S x_{2 n}, v\right) \\
& =\max \left\{1,1, d(z, T v), d^{1 / 2}(z, T v), \frac{d(z, T v)}{2}, \frac{d(z, T v)}{2}, 1\right\} \\
& =d(z, T v) .
\end{aligned}
$$

Hence we have

$$
d(z, T v)=\lim _{n \rightarrow \infty} d\left(S S x_{2 n}, T v\right) \leq[\varphi(d(z, T v))]^{\lambda},
$$

so $T v=z=B v$. Since $B$ and $T$ are weakly compatible, we have $T z=T B v=$ $B T v=B z$.

Next, we prove that $T z=z$. Putting $x=x_{2 n}$ and $y=z$ in inequality $\left(C_{2}\right)$, we have

$$
d\left(S x_{2 n}, T z\right) \leq\left[\varphi\left(M\left(x_{2 n}, z\right)\right)\right]^{\lambda},
$$

where

$$
\begin{aligned}
M\left(x_{2 n}, z\right)= & \max \left\{d\left(A x_{2 n}, B z\right), d\left(A x_{2 n}, S x_{2 n}\right), d(B z, T z),\right. \\
& \left(d\left(A x_{2 n}, T z\right) \cdot d\left(S x_{2 n}, B z\right)\right)^{1 / 2}, \\
& \frac{d\left(A x_{2 n}, T z\right) \cdot d\left(S x_{2 n}, B z\right)}{1+d\left(A x_{2 n}, B z\right)}, \frac{d\left(A x_{2 n}, S x_{2 n}\right) \cdot d(B z, T z)}{1+d\left(A x_{2 n}, B z\right)}, \\
& \left.\frac{1+d\left(A x_{2 n}, T z\right) \cdot d\left(B z, S x_{2 n}\right)}{1+d\left(A x_{2 n}, S x_{2 n}\right) \cdot d(B z, T z)} \cdot d\left(A x_{2 n}, S x_{2 n}\right)\right\} .
\end{aligned}
$$


Taking $n \rightarrow \infty$, we have

$$
\begin{aligned}
\lim _{n \rightarrow \infty} M\left(x_{2 n}, z\right)= & \max \left\{d(z, T z), 1,1, d(z, T z), \frac{d(z, T z) \cdot d(z, T z)}{1+d(z, T z)}\right. \\
& \left.\frac{1}{1+d(z, T z)}, \frac{1+d(z, T z) \cdot d(T z, z)}{2}\right\} \\
= & \frac{1+d^{2}(z, T z)}{2} .
\end{aligned}
$$

This implies that $d(z, T z)=1$ and so $z=T z$. Hence we obtain $z=T z=B z$.

On the other hand, since $z=T z \in T(X) \subset A(X)$, there exist $w \in X$ such that $z=T z=A w$.

Next we prove that $z=S w$. Let $z \neq S w$. Then using $T z=B z=z$ and putting $x=w$ and $y=z$ in $\left(C_{2}\right)$, we have

$$
d(S w, z)=d(S w, T z) \leq[\varphi(M(w, z))]^{\lambda}
$$

where

$$
\begin{aligned}
M(w, z)= & \max \{d(A w, B z), d(A w, S w), d(B z, T z), \\
& (d(A w, T z) \cdot d(S w, B z))^{1 / 2}, \\
& \frac{d(A w, T z) \cdot d(S w, B z)}{1+d(A w, B z)}, \frac{d(A w, S w) \cdot d(B z, T z)}{1+d(A w, B z)}, \\
& \left.\frac{1+d(A w, T z) \cdot d(B z, S w)}{1+d(A w, S w) \cdot d(B z, T z)} \cdot d(A w, S w)\right\} \\
= & \max \left\{1, d(z, S w), 1, d^{1 / 2}(S w, z), \frac{d(z, S w)}{2}, \frac{d(z, S w)}{2}, d(z, S w)\right\} \\
= & d(z, S w) .
\end{aligned}
$$

This implies that $d(S w, z)=1$ and so $S w=z=A w$. Since $A$ and $S$ are compatible, we have $A z=S A w=A S w=S z$ Hence $z=S z=A z=T z=B z$ and hence $z$ is a common fixed point of $A, B, S$ and $T$.

Finally, we prove that $A, B, S$ and $T$ have a unique common fixed point.

Suppose that $w \in X$ is another common fixed point of $A, B, S$ and $T$. Then putting $x=z$ and $y=w$ in inequality $\left(C_{2}\right)$, we have

$$
d(z, w)=d(S z, T w) \leq[\varphi(M(z, w))]^{\lambda},
$$


where

$$
\begin{aligned}
M(z, w)= & \max \{d(A z, B w), d(A z, S z), d(B w, T w) \\
& (d(A z, T w) \cdot d(S z, B w))^{1 / 2} \\
& \frac{d(A z, T w) \cdot d(S z, B w)}{1+d(A z, B w)}, \frac{d(A z, S z) \cdot d(B w, T w)}{1+d(A z, B w)}, \\
& \left.\frac{1+d(A z, T w) \cdot d(B w, S z)}{1+d(A z, S z) \cdot d(B w, T w)} \cdot d(A z, S z)\right\} \\
= & \max \left\{d(z, w), 1,1, d(z, w), \frac{d(z, w) \cdot d(z, w)}{1+d(z, w)}\right. \\
& \left.\frac{1}{1+d(z, w)}, \frac{1+d(z, w) \cdot d(w, z)}{2}\right\} \\
= & \frac{1+d^{2}(z, w)}{2} .
\end{aligned}
$$

This implies that $d(z, w)=1$ and so $z=w$. Therefore, $z$ is a unique common fixed point of $A, B, S$ and $T$.

Similarly if the condition (b) holds, then we obtain the same result.

This completes the proof.

Next we prove the following theorem for compatible mappings of type $(E)$ along with the notion of reciprocally continuous mappings.

Theorem 3.2. Let $A, B, S$ and $T$ be mappings of complete multiplicative metric space $(X, d)$ into itself satisfying $\left(C_{1}\right)$ and $\left(C_{2}\right)$.

Assume that one of the following conditions is satisfied:

(a) $A$ and $S$ are $A$-compatible of type $(E)$ and $A$-reciprocally continuous, $B$ and $T$ are $B$-compatible of type $(E)$ and $B$-reciprocally continuous.

(b) $A$ and $S$ are is $S$-compatible of type $(E)$ and $S$-reciprocally continuous, $B$ and $T$ are $T$-compatible of type $(E)$ and $T$-reciprocally continuous.

Then $A, B, S$ and $T$ a unique common fixed point in $X$.

Proof. By the proof of Theorem 3.1, the sequence $\left\{y_{n}\right\}$ is a multiplicative Cauchy sequence. By the completeness of $X$, the sequence $\left\{y_{n}\right\}$ converges to a point $z \in X$. Consequently the subsequences $\left\{S x_{2 n}\right\},\left\{B x_{2 n+1}\right\},\left\{A x_{2 n}\right\}$ and $\left\{T x_{2 n+1}\right\}$ also converges to $z \in X$, that is,

$$
\lim _{n \rightarrow \infty} S x_{2 n}=\lim _{n \rightarrow \infty} B x_{2 n+1}=\lim _{n \rightarrow \infty} A x_{2 n}=\lim _{n \rightarrow \infty} T x_{2 n+1}=z .
$$


Suppose that $A$ and $S$ are $A$-compatible of type $(E)$ and $A$-reciprocally continuous. Then

$$
A A x_{2 n} \rightarrow S z, \quad A S x_{2 n} \rightarrow S z \quad \text { and } \quad A S x_{2 n} \rightarrow A z
$$

Hence $A z=S z$.

Now, suppose that $z \neq S z$. Then putting $x=z$ and $y=x_{2 n+1}$ in $\left(C_{2}\right)$, we have

$$
d\left(S z, T x_{2 n+1}\right) \leq\left[\varphi\left(M\left(z, x_{2 n+1}\right)\right)\right]^{\lambda},
$$

where

$$
\begin{aligned}
M\left(z, x_{2 n+1}\right) & \max \left\{d\left(A z, B x_{2 n+1}\right), d(A z, S z), d\left(B x_{2 n+1}, T x_{2 n+1}\right),\right. \\
& \left(d\left(A z, T x_{2 n+1}\right) \cdot d\left(S z, B x_{2 n+1}\right)\right)^{1 / 2}, \\
& \frac{d\left(A z, T x_{2 n+1}\right) \cdot d\left(S z, B x_{2 n+1}\right)}{1+d\left(A z, B x_{2 n+1}\right)}, \frac{d(A z, S z) \cdot d\left(B x_{2 n+1}, T x_{2 n+1}\right)}{1+d\left(A z, B x_{2 n+1}\right)}, \\
& \left.\frac{1+d\left(A z, T x_{2 n+1}\right) \cdot d\left(B x_{2 n+1}, S z\right)}{1+d(A z, S z) \cdot d\left(B x_{2 n+1}, T x_{2 n+1}\right)} \cdot d(A z, S z)\right\} .
\end{aligned}
$$

Taking $n \rightarrow \infty$, we have

$$
\begin{aligned}
& \lim _{n \rightarrow \infty} M\left(z, x_{2 n+1}\right) \\
& =\max \left\{d(S z, z), 1,1, d(S z, z), \frac{d(S z, z) \cdot d(S z, z)}{1+d(S z, z)}\right. \\
& \left.\quad \frac{1}{1+d(S z, z)}, \frac{1+d(S z, z) \cdot d(z, S z)}{2}\right\} \\
& =\frac{1+d^{2}(z, S z)}{2}
\end{aligned}
$$

Hence we have

$$
d(S z, z) \leq\left[\varphi\left(\frac{1+d^{2}(z, S z)}{2}\right)\right]^{\lambda} \leq d^{\lambda}(z, S z)
$$

which implies that $S z=z$. Hence $z=A z=S z$. Since $S(X) \subset B(X)$, there exists $u \in X$ such that $z=S z=B u$.

Let $T u \neq B u$. Then by putting $x=z$ and $y=u$ in $\left(C_{2}\right)$, we have

$$
d(B u, T u)=d(S z, T u) \leq[\varphi(M(z, u))]^{\lambda},
$$


where

$$
\begin{aligned}
M(z, u)= & \max \{d(A z, B u), d(A z, S z), d(B u, T u) \\
& d(A z, T u) \cdot d(S z, B u))^{1 / 2}, \\
& \frac{d(A z, T u) \cdot d(S z, B u)}{1+d(A z, B u)}, \frac{d(A z, S z) \cdot d(B u, T u)}{1+d(A z, B u)}, \\
& \left.\frac{1+d(A z, T u) \cdot d(B u, S z)}{1+d(A z, S z) \cdot d(B u, T u)} \cdot d(A z, S z)\right\} \\
= & \max \left\{1,1, d(B u, T u), d^{1 / 2}(B u, T u), \frac{d(B u, T u)}{2}, \frac{d(B u, T u)}{2}, 1\right\} \\
= & d(B u, T u) .
\end{aligned}
$$

Hence $B u=T u=z$. Since $B$ and $T$ are $B$-compatible of type $(E)$ and $B$ reciprocally continuous, by Proposition 2.5, we obtain $B z=B T u=T B u=T z$.

Moreover putting $x=z$ and $y=z$ in $\left(C_{2}\right)$, we have

$$
d(z, T z)=d(S z, T z) \leq[\varphi(M(z, z))]^{\lambda},
$$

where

$$
\begin{aligned}
M(z, z)= & \max \{d(A z, B z), d(A z, S z), d(B z, T z), \\
& (d(A z, T z) \cdot d(S z, B z))^{1 / 2}, \\
& \frac{d(A z, T z) \cdot d(S z, B z)}{1+d(A z, B z)}, \frac{d(A z, S z) \cdot d(B z, T z)}{1+d(A z, B z)}, \\
& \left.\frac{1+d(A z, T z) \cdot d(B z, S z)}{1+d(A z, S z) \cdot d(B z, T z)} \cdot d(A z, S z)\right\} \\
= & \max \left\{d(z, T z), 1,1, d(z, T z), \frac{d(z, T z) \cdot d(z, T z)}{1+d(z, T z)},\right. \\
& \left.\frac{1}{1+d(z, T z)}, \frac{1+d(z, T z) \cdot d(T z, z)}{2}\right\} \\
= & \frac{1+d^{2}(z, T z)}{2} .
\end{aligned}
$$

Hence we have

$$
d(z, T z) \leq\left[\varphi\left(\frac{1+d^{2}(z, T z)}{2}\right)\right]^{\lambda} \leq d^{\lambda}(z, T z)
$$


which implies that $T z=z$. Hence $z=A z=S z=B z=T z$, that is, $z$ is a common fixed point of $A, B, S$ and $T$.

Similarly, we can prove $z$ is a common fixed point of $A, B, S$ and $T$ if the condition (b) holds.

The uniqueness easily follows from $\left(C_{2}\right)$. This complete the proof.

\section{References}

[1] M. Abbas, B. Ali, Y.I. Suleiman, Common fixed points of locally contractive mappings in multiplicative metric spaces with application, Int. J. Math. Math. Sci., 2015 (2015), Article ID 218683, 7 pages, doi: 10.1155/2015/218683.

[2] A.E. Bashirov, E.M. Kurplnara, A. Ozyapici, Multiplicative calculus and its applications, J. Math. Anal. Appl., 337 (2008), 36-48, doi: 10.1016/j.jmaa.2007.03.081.

[3] S.M. Kang, P. Kumar, S. Kumar, B.Y. Lee, Common fixed points for compatible mappings of types in multiplicative metric spaces, Int. J. Math. Anal., 9 (2015), 1755-1767, doi: 10.12988/ijma.2015.53104.

[4] S.M. Kang, P. Kumar, S. Kumar, P. Nagpal, S.K Garg, Common fixed points for compatible mappings and its variants in multiplicative metric spaces, Int. J. Pure Appl. Math., 102 (2015), 383-406, doi: 10.12732/ijpam.v102i2.14.

[5] M. Özavsar, A.C. Çevikel, Fixed points of multiplicative contraction mappings on multiplicative metric spaces, ArXiv: 1205.5131v1 [math.GM] (2012), 14 pages.

[6] R.P. Pant, A common fixed point theorem under a new condition, Indian. J. Pure Appl. Math., 30 (1999), 147-152.

[7] M.R. Singh, Y.M. Singh, On various types of compatible maps and common fixed point theorems for non-continuous maps, Hacettepe J. Math. Statist., 40 (2011), 503-513. 
